\title{
On Cochlear Impedances and the Miscomputation of Power Gain
}

\author{
Christopher A. Shera, ${ }^{1}$ Elizabeth S. Olson, ${ }^{2}$ and John J. Guinan Jr. ${ }^{1}$ \\ ${ }^{1}$ Eaton-Peabody Laboratories, Massachusetts Eye and Ear Infirmary, 243 Charles Street, Boston, MA 02114, USA \\ ${ }^{2}$ Department of Otolaryngology, Head and Neck Surgery, Columbia University, New York, NY 10032, USA
}

Received: 6 June 2011; Accepted: 9 August 2011; Online publication: 27 September 2011

\begin{abstract}
In their article, "Measurement of cochlear power gain in the sensitive gerbil ear," Ren et al. (Nat Commun 2:216, 2011) claim to provide "the first direct experimental evidence of power amplification in the sensitive living cochlea." While we recognize the technical challenges of the experiments and appreciate the beauty of the data, the authors' analysis and interpretation of the measurements are invalid. We review the concept of impedance (i.e., the ratio of pressure to velocity) as it applies to cochlear mechanics and show that Ren et al. mistakenly equate the impedances near the basilar membrane and stapes with the impedance characteristic of an infinite, uniform tube of fluid. As a consequence of this error, Ren et al.'s measurements and analysis provide no evidence for power amplification in the cochlea. Compelling evidence for power amplification has, however, been previously provided by others.
\end{abstract}

Keywords: cochlea, cochlear amplifier, power gain, impedance

\section{INTRODUCTION}

Electrohydromechanical processes within the mammalian inner ear collectively amplify the soundinduced motion of the cochlear partition, thereby enhancing the sensitivity, frequency selectivity, and, perhaps most importantly, the dynamic range of hearing (reviewed in Dallos 1996). Theoretical analyses suggest that the mammalian cochlear amplifier

Correspondence to: Christopher A. Shera - Eaton-Peabody Laboratories · Massachusetts Eye and Ear Infirmary $\cdot 243$ Charles Street, Boston, MA 02114, USA. shera@epl.meei.harvard.edu operates by boosting the power carried by the pressure waves propagating within it (Neely 1980; Zweig 1991; de Boer 1995; Shera 2007). However, the active enhancement of cochlear vibration amplitudes does not, in principle, require cycle-by-cycle power amplification. Displacement enhancement could arise, for example, via mechanisms similar to mechanical transformers (e.g., levers) or by reducing the impedance (e.g., stiffness) of the vibrating structures (e.g., Kolston 1988). Although cochlear power amplification is biologically feasible-direct measurements have established that the bundles of bullfrog saccular hair cells are capable of doing mechanical work (Martin and Hudspeth 1999) - the biophysical processes subserving amplification in the mammalian cochlea evidently differ from those operating in the bullfrog sacculus, at least at high frequencies. In particular, the enhancement of basilar-membrane motion in the mammalian cochlea appears to depend primarily on forces produced by outer hair cell soma rather than by the bundles (Liberman et al. 2002). Thus, determining whether or not the mammalian cochlea provides actual power (as opposed to displacement) amplification - and if so, how much-has been a central question in auditory biophysics.

In a recent report, Ren, He, and Gillespie (2011) claim to resolve this issue by providing "the first direct experimental evidence of power amplification in the sensitive living cochlea." Since the measurement of power is technically difficult, especially in vivo, Ren et al.'s results, if substantiated, would be an experimental and analytical tour de force worthy of publication in a high-profile journal. Unfortunately, and notwithstanding the merits of the data, a careful reading of the paper reveals that the authors' analysis and interpretation are invalid. In fact, the measurements provide no evidence whatsoever for power amplification in the cochlea. 


\section{CRITIQUE}

Our critique of Ren et al.'s analysis and conclusions has two main parts. In the first part, we identify a major conceptual problem underlying their analysis and point out a serious technical error in their estimates of energy and power gain. In the second part, we consider whether Ren et al.'s metric might nevertheless provide a useful assay of cochlear power amplification.

\section{A problem with impedance}

Power is force times velocity, and it is important to recognize at the outset that Ren et al. measured only displacement, not power. Contrary to their claims, the authors therefore provide no direct evidence for anything involving power, which would require simultaneous measurements of both velocity (as obtained from displacement in their study) and force or pressure (which they did not measure). Lacking direct measurements of force or pressure, Ren et al. must convert measurements of velocity into power. For this, the relevant impedance must be known.

\section{A few remarks about impedance}

Impedance is a physical concept that relates force to velocity, or in acoustics, pressure to velocity. Here, we consider the acoustic case and define impedance as pressure divided by velocity. In certain simple situations, such as in free space or at the driving end of a tube or horn of standard mathematical shape, the impedance has been determined analytically and can be looked up in a book (e.g., Rayleigh 1896). For plane waves in free space, or in an infinite lossless tube, the impedance is $\rho c$, the product of the mass density of the medium, $\rho$, and the speed of sound, $c$. Because these systems are uniform (homogeneous) and infinite, the impedance is constant everywhere. The impedance $\rho c$ is also real and positive. This tells us that sound energy is absorbed by the medium (i.e., that it radiates away to infinity). For a closed tube, by contrast, the impedance is equal to $\rho c$ multiplied by an imaginary factor whose value depends both on frequency and on the length of the tube. As the frequency is changed, the magnitude of the multiplicative factor varies between zero and infinity, reflecting the fact that at some frequencies the pressure, and at others the particle velocity, becomes zero at the point of measurement. Thus, for the closed tube the impedance at any given location varies with frequency, and conversely, at any given frequency varies with location. The impedance is also purely imaginary, rather than real, which means that energy is neither absorbed nor radiated away. This makes sense; since the sound is reflected at the end and cannot escape to infinity, energy is "trapped" or stored within the tube.
Now, consider impedances and power flow within the cochlea. At the macromechanical level considered here, the relevant velocity is that of the cochlear partition, and the relevant pressure is that at the surface of the basilar membrane-or, indeed, the pressure difference across the basilar membrane, for it is this force that moves the partition up and down, stimulating the sensory cells (Voss et al. 1996; Olson 2001; Dong and Olson 2009). Given the cochlea's convoluted, three-dimensional structure, and the complexity of its myriad mechanical components, it should come as no surprise that impedances in the cochlea differ substantially from those in tubes and horns. Although tubes have no internal parts to interact with the sound they carry, the structures embedded within the cochlea's fluid medium-the basilar membrane and organ of Corti-have a profound effect on the mechanical response. When the stapes or any of the cochlea's constituents vibrates, the motion produces forces, communicated through the fluid, that cause the other structures within the cochlea to move or deform. Indeed, forces produced by outer hair cells couple back to the stapes and therefore even result in the movement of structures outside the cochlea (e.g., otoacoustic emissions). Thus, at any given frequency and location, the quantitative relationship between the pressure and the basilarmembrane (BM) velocity - the impedance-depends on a complex network of moving parts and geometrical relationships. As a result, impedances in the cochlea are much more complicated than in infinite tubes, and their values are not so easily computed.

Although impedances express the relationship between the local pressure and the local velocity, they depend not only on the local properties of the medium, but on everything the medium is coupled to, directly or indirectly. In an infinite, homogeneous medium, plane waves propagate freely, uncoupled to any other structures, and the corresponding impedance therefore has the constant value $\rho c$. In the cochlea, however, the fluid is strongly coupled to many other things; as a result, cochlear impedances vary both with frequency and with location. They depend not only on the characteristics of the fluid but also on the size and shape of the cochlea, on the mechanical properties of the cochlear partition (e.g., its mass, stiffness, and active forces), and on the load presented to the cochlea by the middle ear and beyond. Although impedances measured in the fluid near the BM are sometimes referred to as "fluid impedances" in the literature (e.g., Steele and Taber 1979), they depend on far more than just the fluid.

\section{Misidentification of the relevant impedances}

In their analysis of cochlear power flow, Ren et al. ignore the observations about impedance reviewed 
above and assume that the relevant impedance in the cochlea is everywhere $\rho c$, the impedance of a plane wave propagating in an infinite, homogeneous medium (e.g., in an infinite fluid-filled tube). As we have indicated, this assumption is wrong, for two reasons. First, the cochlea is not infinite in extent. Indeed, the cochlear fluid and the sound waves propagating within it are confined to a finite chamber whose dimensions are small compared to the wavelength of sound. Just as the impedance of a pipe closed at one end differs from that of an infinite tube (Fletcher 1992), so the boundary conditions at the cochlear walls have a significant influence on cochlear impedances (Mammano and Nobili 1993).

Second, the cochlea is not homogeneous; it has an internal structure that enables it to function as a sensory transducer. As a result, the impedances relevant to the computation of power depend not only on the fluid and the walls, but also on the properties of the cochlear partition, the boundary conditions at the middle ear, and so on. At the stapes, for example, the effective impedance is influenced by the stiffness of the basilar membrane-the more compliant the membrane, the easier it is to move the stapes and the lower the impedance. As a result, the relevant impedance at the stapes (the cochlear input impedance) has a value very different from the specific acoustic impedance of the cochlear fluid, as demonstrated both by theory and by direct measurement (Lynch et al. 1982; Rosowski et al. 1986; Shera and Zweig 1991).

Although Ren et al. correctly note that classical cochlear models assume that hydromechanical energy is propagated through the cochlear fluids, they ignore what those models say about where and how the energy is injected (e.g., via electromechanical transduction in the outer hair cells) and absorbed. When they employ the specific acoustic impedance of the fluid and ignore the properties of the cochlear partition, Ren et al. effectively assume that acoustic energy, once introduced into the fluid, propagates away to infinity. The cochlear models to which Ren et al. refer, however, indicate that this is not the case. Instead, energy transferred to the fluid in one region, either from the stapes or from the organ of Corti, is absorbed by the partition in another (e.g., near the peak of the wave). The models indicate that the direction of time-averaged power transfer to or from the traveling wave is controlled by the sign of the real part of the impedance of the cochlear partition. This impedance depends, among a great many other things, on the stiffness of the basilar membrane and the magnitudes and phases of the forces produced by outer hair cells. Consequently, the relevant impedance varies with frequency and location within the cochlea; the impedance seen by the stapes is not the same as the impedance seen by the basilar membrane near the peak of the traveling wave. By using the specific acoustic impedance of the fluid at all frequencies and at all locations, Ren et al. ignore the role of the cochlear partition in energy transfer and seriously distort the physics underlying the models they cite. To suggest that the relevant cochlear impedance is $\rho c$, as Ren et al. do, is to regard the cochlea as an infinite tube containing no basilar membrane, no organ of Corti, and no hair cells-indeed, containing nothing but salty water. One's ears can only be deaf to such a proposition.

\section{Technical error in the analysis}

In addition to misidentifying the relevant impedances, Ren et al. make a technical error that renders their analysis dimensionally incorrect and therefore logically flawed at the most basic level. In particular, Ren et al. compute power using an incorrect formula. To convert measurements of volume velocity (which they denote $V$ ) into power (which they denote $I$ ), Ren et al. use the formula $I=\rho c V^{2}$, where $\rho c$ is the specific acoustic impedance of the medium (e.g., the cochlear fluid). But $I$ defined in this way has dimensions of power $\times$ area, not of power. To see this, note that if M, $\mathrm{L}$, and $\mathrm{T}$ represent, respectively, the dimensions of mass, length, and time, then the dimensions of $\rho c V^{2}$, denoted $\left[\rho c V^{2}\right]$, are

$[\rho][c][V]^{2}=\left(\frac{\mathrm{M}}{\mathrm{L}^{3}}\right)\left(\frac{\mathrm{L}}{\mathrm{T}}\right)\left(\frac{\mathrm{L}^{3}}{\mathrm{~T}}\right)^{2}=\left(\frac{\mathrm{ML}^{2}}{\mathrm{~T}^{3}}\right) \mathrm{L}^{2}=[$ power $][$ area $]$,

where the dimensions of power are those of energy $(E)$ per time, and energy has the dimensions of mass times velocity squared $\left([E]=\mathrm{ML}^{2} / \mathrm{T}^{2}\right)$. As a result of this dimensional discrepancy, Ren et al.'s calculations of the "energy input and output functions of the basilar membrane" (obtained by integrating the formula for $I$ over some unspecified time interval) do not have units of joules (J), as indicated on the ordinate of their Fig. $5 \mathrm{~b}$, but of $\mathrm{J} \cdot \mathrm{m}^{2}$. Although Ren et al. refer to the value given by their formula as "the sound energy passing through [a certain area] ... in $1 \mathrm{~s}$," the quantity they compute actually has no clear physical interpretation.

The formula used by Ren et al. to compute "power" is typographically but not semantically identical to a standard equation for power flow in acoustics, namely $I=\rho c V^{2}$ (e.g., Kinsler et al. 1982). In the standard equation, $I$ is the acoustic intensity (timeaveraged power per unit area) carried by a plane wave propagating in an infinite medium of specific acoustic impedance $\rho c$, and $V$ is the corresponding root mean square (rms) acoustic particle velocity. When the 
variables $I$ and $V$ are thus correctly interpreted, the equation is, of course, dimensionally consistent. But Ren et al.'s substitution of a volume-velocity amplitude for an rms particle velocity, perhaps facilitated by use of the symbol $V$ for both, renders the equation both dimensionally inconsistent and physically meaningless.

\section{An ad hoc metric unrelated to power gain}

Despite these basic physical errors in the analysis, the reader may wonder whether the value of the gain metric introduced by Ren et al. might nevertheless be indicative of cochlear amplification. Using their data, Ren et al. are able to compute a quantity, $G_{\mathrm{e}}$, that they call the energy (or power) gain of the basilar membrane. As they explain in the "Methods," Ren et al. interpret $G_{\mathrm{e}}$ as the ratio of the power associated with basilar-membrane vibration near the peak of the wave to the power input at the stapes. Empirical values of $G_{\mathrm{e}}$ greater than one, they argue, indicate that the cochlear amplifier has boosted the power carried by the traveling wave, establishing that the cochlea provides power amplification. Although their physical derivation is clearly flawed (see above), Ren et al.'s demonstration of power gain might still be logically viable: If values of $G_{\mathrm{e}}>1 \mathrm{do}$, in fact, characterize systems with positive power gain, as Ren et al. claim, then their empirical finding that $G_{\mathrm{e}}$ can be greater than 1 would indeed establish, albeit somewhat fortuitously, that the mammalian cochlea provides power amplification.

Figure 1 demonstrates, however, that values of $G_{\mathrm{e}}>1$ can arise even in a completely passive structure. The figure shows values of $G_{\mathrm{e}}$ computed for a simple, passive model of the cochlea (e.g., Zweig et al. 1976) as a function of the effective damping constant of the cochlear partition. For the results shown in the figure, the damping

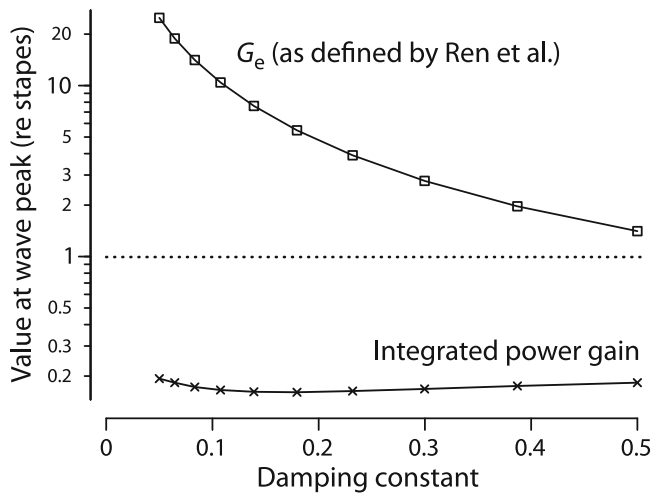

FIG. 1. Two metrics computed in a passive cochlear model as a function of the assumed damping constant. The physically based gain metric involving integrated power $(x)$ is always less than 1 , correctly indicating the absence of cochlear amplification in the model. By contrast, the metric $G_{\mathrm{e}}$ proposed by Ren et al. (white square) does not measure power gain; its value can be significantly greater than 1 , even in a passive system. constant was everywhere positive and the model therefore entirely passive. Although the cochlear partition in the model always attenuates the power carried by the traveling wave and never amplifies it, the figure demonstrates that the values of $G_{\mathrm{e}}$ can be significantly greater than 1. (The parameters of the model can also be adjusted to yield values of $G_{\mathrm{e}}$ less than 1.) Thus, Ren et al.'s finding that empirical values of $G_{\mathrm{e}}$ are generally greater than 1 provides no evidence of cochlear power gain. Despite its name, $G_{\mathrm{e}}$ does not measure gain associated with energy or power. For comparison, the figure also shows corresponding values of a physically based metric of net power gain proposed previously (de Boer and Nuttall 2001; Shera 2007). The metric involves the total power carried by the wave at the peak of its envelope normalized by the input power. Computing its value involves integrating the power transferred to or from the organ of Corti between the stapes and the peak of the wave. As expected for a passive system, the power gain measured in this way is always less than 1 .

\section{What determines the value of $G_{e}$ ?}

Because of the physical errors outlined above, Ren et al.'s "power" gain $G_{\mathrm{e}}$ is nothing but the square of a volume-velocity gain $\left(G_{\mathrm{e}}=G_{\mathrm{vol}}^{2}\right)$. In general, volumevelocity gains are in no way equivalent to power gains. It is easy to construct acoustical-mechanical systems that manifest significant volume-velocity gains while having power gains less than 1 (i.e., energy losses). For example, volume-velocity gains greater than $1 \mathrm{can}$ occur in passive acoustic transformers, such as horns (Fletcher 1992). The middle ear driven in reverse provides another example, this time from the auditory periphery (Puria 2003). In the cochlea, the fact that Ren et al.'s ad hoc metric of basilar-membrane "power" or volume-velocity gain (re stapes) can be greater than 1 even in the absence of amplification can be understood as the consequence of conservation of fluid mass and the fact that BM traveling waves contain multiple wavelengths. Because the cochlear fluids are nearly incompressible and a negligible amount of fluid flows through the helicotrema at the stimulus frequency employed, the total instantaneous volume displacement of the $\mathrm{BM}$, integrated along its length, must equal the volume displacement of the stapes. But the integrated BM volume displacement is the sum of contributions that alternate polarity every half wavelength as the BM displacement alternates between positive and negative ("up" and "down") along its length. Figure 1c in Ren et al.'s paper, which shows a snapshot of the BM traveling wave as it appears at a particular moment of time, illustrates that the volume displacements of adjacent half-wavelength regions alternate in sign. As a consequence of this alternation, the volume 
displacements associated with different regions largely cancel one another when added together, and the total volume displacement of the BM can therefore be much smaller than the volume displacement of any given section. Thus, if one chooses to compute the instantaneous volume displacement of some particular BM section at some particular moment-such as the one rather arbitrarily selected by Ren et al. near the peak of the wave-one can readily find that the volume displacement of the chosen BM section is larger than the total volume displacement of the BM and hence also larger than that of the stapes. In other words, Ren et al.'s metric $G_{\mathrm{e}}$ of BM volume-velocity gain (re stapes) can be greater than 1, even in a passive cochlea. Simply put, $G_{\mathrm{e}}$ provides an arbitrary measure of volume-velocity gain but does not quantify power gain. ${ }^{1}$

Of course, the exact value of $G_{\mathrm{e}}$ one obtains will depend on the location and spatial extent of the particular BM section one chooses to analyze. It will also depend on the instantaneous wave pattern of the traveling wave in that region, a pattern that can be modified by cochlear amplification and can vary with stimulus intensity. The crucial point, however, is that with an advantageous choice of moving boundary, volume-velocity gains greater than 1 can, in principle, occur under entirely passive conditions, as is easily verified in cochlear models (see Fig. 1). Thus, although Ren et al.'s measurements and analysis corroborate previous studies showing that cochlear responses are nonlinear, they provide no evidence whatsoever for actual power amplification.

\section{PREVIOUS DISCUSSIONS OF COCHLEAR POWER GAIN}

Although Ren et al.'s published analysis does not meaningfully address the issue, previous studies have reported and discussed estimates of the power gain of the cochlear amplifier. These estimates have been rigorously derived both by analyzing $\mathrm{BM}$ measurements within the context of hydromechanical models (Zweig 1991; Brass and Kemp 1993; de Boer and Nuttall 2001; Shera 2007) and by combining direct measurements of $\mathrm{BM}$ velocity and intracochlear pressure (Olson 2001; Dong and Olson 2009). Some of these studies have already convincingly established

\footnotetext{
1 Physically correct definitions of cochlear power gain involve averaging over a stimulus cycle and integrating the power flow (i.e., acoustic intensity) along the entire extent of the wave (de Boer and Nuttall 2001; Shera 2007). The computation requires knowledge of the real part of the relevant impedances (i.e., the impedances at the $\mathrm{BM}$ and at the stapes).
}

that the mammalian cochlea provides power amplification to boost the sensitivity and dynamic range of hearing. Going beyond this qualitative statement to obtain reliable quantitative estimates of the power gain of the amplifier remains challenging. The principal difficulty resides in making accurate simultaneous measurements of both pressure and velocity and/or in deriving credible theoretical estimates of these quantities from other measurements. In their report, Ren et al. would have us believe that they have discovered a significant shortcut for this challenging problem. They have not.

\section{ACKNOWLEDGMENTS}

This work was supported by grants R01 DC003687 (CAS), DC003130 (ESO), and DC00235 (JJG) from the NIDCD, National Institutes of Health.

\section{REFERENCES}

Brass D, Kemp DT (1993) Analyses of Mössbauer mechanical measurements indicate that the cochlea is mechanically active. J Acoust Soc Am 93:1502-1515

Dallos P (1996) Overview: cochlear neurobiology. In: Dallos P, Popper AN, Fay RR (eds) The cochlea. Springer, New York, pp 1-43

DE BOER E (1995) The 'inverse problem' solved for a three-dimensional model of the cochlea I. Analysis. J Acoust Soc Am 98:896-903

De Boer E, Nuttall AL (2001) Power gain of the cochlear amplifier. In: Breebaart DJ, Houtsma AJM, Kohlrausch A, Prijs VF, Schoonhoven R (eds) Physiological and psychological bases of auditory function. Shaker, Maastricht, pp 1-7

Dong W, Olson ES (2009) In vivo impedance of the gerbil cochlear partition at auditory frequencies. Biophys J 97:1233-1243

Fletcher NH (1992) Acoustic systems in biology. Oxford Univ. Press, New York

KinsLer LF, Frey AR, Coppens AB, SANDers JV (1982) Fundamentals of acoustics. Wiley, New York

Kolston PJ (1988) Sharp mechanical tuning in a cochlear model without negative damping. J Acoust Soc Am 83:1481-1487

Liberman MC, Gao J, He DZZ, Wu X, Jia S, Zuo J (2002) Prestin is required for electromotility of the outer hair cell and for the cochlear amplifier. Nature 419:300-304

Lynch TJ, Nedzelnitsky V, Peake WT (1982) Input impedance of the cochlea in cat. J Acoust Soc Am 72:108-130

Mammano F, Nobili R (1993) Biophysics of the cochlea: linear approximation. J Acoust Soc Am 93:3320-3332

Martin P, Hudspeth AJ (1999) Active hair-bundle movements can amplify a hair cell's response to oscillatory mechanical stimuli. Proc Natl Acad Sci USA 96:14,306-14,311

NEELY ST (1980) Backward solution of a two-dimensional cochlear model. J Acoust Soc Am 67:S75

OLSON ES (2001) Intracochlear pressure measurements related to cochlear tuning. J Acoust Soc Am 110:349-367

PURIA S (2003) Measurements of human middle ear forward and reverse acoustics: implications for otoacoustic emissions. J Acoust Soc Am 113:2773-2789

Rayleigh JW (1896) Theory of sound. Dover, New York

Ren T, He W, Gillespie PG (2011) Measurement of cochlear power gain in the sensitive gerbil ear. Nat Commun 2:216 
Rosowski JJ, Carney LH, Lynch TJ, Peake WT (1986) The effectiveness of external and middle ears in coupling acoustic power into the cochlea. In: Allen JB, Hall JL, Hubbard A, Neely ST, Tubis A (eds) Peripheral auditory mechanisms. Springer, Berlin, pp 56-59

SHERA CA (2007) Laser amplification with a twist: traveling-wave propagation and gain functions from throughout the cochlea. J Acoust Soc Am 122:2738-2758

Shera CA, Zweig G (1991) A symmetry suppresses the cochlear catastrophe. J Acoust Soc Am 89:1276-1289
Steele CR, TABer LA (1979) Comparison of WKB calculations and experimental results for threedimensional cochlear models. J Acoust Soc Am 65:1007-1018

Voss SE, Rosowski JJ, Peake WT (1996) Is the pressure difference between the oval and round window the effective stimulus for the cochlea? J Acoust Soc Am 100:1602-1616

ZweIG G (1991) Finding the impedance of the organ of Corti. J Acoust Soc Am 89:1229-1254

Zweig G, Lipes R, Pierce JR (1976) The cochlear compromise. J Acoust Soc Am 59:975-982 